$63^{\text {ème }}$ Congrès de la SFCO, 03035 (2015)

DOI:10.1051/sfco/20156303035

(C) Owned by the authors, published by EDP Sciences, 2015

\title{
Gestion des séquelles d'une complication de greffe sinusienne : à propos d'un cas
}

\author{
Bornert $\mathrm{F}^{*, * *}$, Bridonneau $\mathrm{T}^{*, * *}$, Eglin $\mathrm{F}^{* * *}$ \\ *Unité de Chirurgie buccale, Hôpitaux Universitaires de Strasbourg \\ **Faculté de Chirurgie-Dentaire de Strasbourg \\ ***Pratique privée, Belfort
}

La technique d'élévation sinusienne par voie latérale a été décrite dès les années 80 par Boyne et Tatum s'est très largement diffusée dans le monde depuis aboutissant au consensus de Boston en 1996 (Jensen et al., 1998).

Le comblement du sinus avec particules de comblement constitue ainsi le gold standard. Cependant, il subsiste des inconvénients tels que la morbidité du site donneur ou encore le coût des biomatériaux ou certaines complications. Les infections peuvent toucher le sinus ou le biomatériaux. C'est une complication qui met en jeu le pronostic de la greffe. Elles sont d'origines différentes : sinusienne, dentaire, systémique ou lié à une faute d'asepsie. La prévalence des infections est de 2,9 \% selon Pjetrusson et al. (2008).

Nous décrivons ici le cas d'un patient qui présentait initialement une communication bucco-sinusienne persistante suite à l'extraction de la dent $\mathrm{n}^{\circ} 16$. Il a été proposé de greffer le sinus en complément de la gestion de la CBS afin de compenser l'édentement 14 à 17. Un prélèvement rétro-molaire avait été réalisé afin de greffer le sinus mais plusieurs épisodes infectieux ont à de multiples interventions et la fonte du greffon sur une période de 2 ans.

Au moment où le patient a été adressé pour une prise en charge, celui-ci ne présente plus de CBS mais un raccourcissement complet du vestibule supérieur droit suite la réalisation d'un lambeau de glissement vestibulaire se fixant au delà du milieu de la crête édentée. Il y absence quasi-complète de la paroi latérale du sinus au niveau du secteur édenté. Le patient était très gêné et la réalisation d'une prothèse amovible par le praticien traitant.

La prise en charge s'est déroulée en deux temps chirurgicaux. Une dissection en épaisseur partielle du lambeau vestibulaire a été faite pour le suturer apicalement en complément d'une greffe épithélioconjonctive et une cicatrisation dirigée par une plaque palatine avec retour vestibulaire. Après quelques mois, malgré un gain évident de vestibulaire un inconfort persistait. Une nouvelle intervention, similaire à la première a été réalisée rendant possible le port d’une prothèse amovible d'usage après une période d'observation de 6 mois. Le patient n'était plus motivé pour entreprendre une nouvelle chirurgie pré-implantaire.

Ce cas clinique rappelle que la technique d'élévation sinusienne n'est pas anodine. La technique chirurgicale mise en œuvre ici rappelle celle de l'approfondissement de vestibule associé à une greffe épithélio-conjonctive et à une technique de cicatrisation dirigée. Une difficulté supplémentaire était constituée par la destruction quasi-complète de la paroi latérale du sinus maxillaire. D’autre part, du fait d'une insuffisance de suivi et de compliance du patient, la prise en charge nécessita un deuxième temps chirurgical.

This is an Open Access article distributed under the terms of the Creative Commons Attribution License 4.0, which permits unrestricted use, distribution, and reproduction in any medium, provided the original work is properly cited. 
$63^{\text {ème }}$ Congrès de la SFCO

Nom et adresse du conférencier

Fabien BORNERT

Département de Chirurgie Buccale, Hôpitaux Universitaires de Strasbourg

Pôle de Médecine et de Chirurgie Bucco-Dentaires

Hôpital Civil CHRU Strasbourg

67000 Strasbourg (France)

fabien.bornert@chru-strasbourg.fr 